\title{
The association between cancer treatments and oral diseases
}

\author{
Abstracted from \\ Hong CH, Napeñas JJ, Hodgson BD, et al. \\ A systematic review of dental disease in patients undergoing cancer therapy. \\ Support Care Cancer 2010; 18: 1007-1021. \\ Address for correspondence: Catherine Hong, Department of Oral Medicine, \\ Carolinas Medical Center, 1000 Blythe Blvd, P.O. Box 32861, Charlotte, NC 28232, USA. \\ E-mail: catherine.hong@carolinashealthcare.org
}

\section{Question: How do cancer treatments affect oral health?}

Data sources MEDLINE/PubMed and EMBASE

Study selection The review included papers published between January 1, 1990 and December 31, 2008. The primary outcome was to retrieve all literature containing original data on dental caries, periodontal disease and pre-cancer dental clearance protocols in cancer patients undergoing head and neck radiotherapy, chemotherapy or combined treatment modalities. Excluded studies included systematic and non-systematic reviews, microbiology studies, growth and development studies, organ transplant studies, studies eliciting dental complications through questionnaires, studies reporting data from previous publications, phase I and II trials, opinion papers, case reports, articles published before 1990, and publications from the 1990 National Cancer Institute Monographs, which were based on the 1989 National Institutes of Health Development Consensus Conference on the Oral Complications of Cancer Therapies.

Data extraction and synthesis Each article was independently evaluated by two reviewers with pilot-tested collection forms customised for reviewing dental disease data. Dental caries was assessed by the presence $(\mathrm{Y} / \mathrm{N})$, DMFT/dmft (decayed, missing, and filled teeth: DMFT for permanent adult teeth and dmft signifying deciduous teeth), and DMFS/dmfs indexes (decayed, missing, and filled surfaces: DMFS for permanent adult teeth and dmfs signifying deciduous teeth), if available. In addition, periodontal health was assessed using the plaque and gingival indexes. Further data collected for each article such as type of study, blinding, presence of control group, scale validity, and sample size were used to determine quality outcomes utilised to determine the weighted prevalence of caries and dental infection.

Results Sixty-four published papers between 1990 and 2008 were reviewed. The weighted overall prevalence of dental caries was $28.1 \%$. The overall DMFT for patients who were post-antineoplastic therapy was $9.19(n=457)$. The overall plaque index for patients who were post-antineoplastic therapy was $1.38(n=189)$. The gingival index for patients who were post-chemotherapy was $1.02(n=162)$. The weighted prevalence of dental infection/abscess during chemotherapy was reported in three studies and was 5.8\%.

Conclusions Patients who were post-radiotherapy had the highest DMFT. The use of fluoride products and chlorhexidine rinses is beneficial in patients who are post-radiotherapy. There continues to be a lack of clinical studies on the extent and severity of dental disease that are associated with infectious complications during cancer therapy.

\section{Commentary}

Surgical resection, radiation and chemotherapy, either used alone or in combination, are the three most common modalities of cancer treatment. Although these modalities are effective in eradicating the tumour, they may produce unavoidable damage to oral tissues and the dental apparatus, especially in the case of head and neck cancer. Oral complications may occur during and following cancer therapy. The mucosal lining of the gastrointestinal tract, including the oral mucosa, is a prime target for treatment-related toxicity due to its rapid cell turnover rate. Chemotherapy, in general, and radiation to the head and neck regions often impair cell division and disrupt normal replacement of oral mucosa. In addition, localised head and neck radiotherapy can result in structural and functional alterations of underlying supportive tissues, including salivary glands and bone. Furthermore, surgical treatments of head and neck cancers may be disfiguring and reconstruction efforts may be futile.

The purpose of this systematic review was to evaluate the literature and update the current understanding of the impact of cancer therapies on the teeth and periodontium, as well as the role of pre-cancer dental treatments since the 1989 National Institutes of Health Development Consensus Conference on the Oral Complications of Cancer Therapies. ${ }^{1}$ Due to the cumulative nature of medical research, new knowledge has surely accumulated in this area since the statement was initially prepared. Thus, this review is very relevant and informative.

The review had rigorous inclusion and exclusion criteria for both observational and interventional study designs. Of the 64 studies included, 46 were observational and 18 interventional. Furthermore, 31 studies recruited adult patients, 24 recruited paediatric patients, 4 included both paediatric and adult patients and 5 did not provide the age of the population sampled. The two most common malignancies in the review were head and neck cancer (37 studies) and haematological malignancies (31 studies).

The weighted overall prevalence of dental caries was $28.1 \%$ from 19 observational studies. Interestingly, the weighted prevalence of dental caries was highest in patients who received only chemotherapy compared to those who received radiotherapy or chemo-radiotherapy. This may be attributed to more aggressive dental management of patients prior to radiotherapy compared to those being prepared for chemotherapy. Patients undergoing head and neck radiotherapy have a high risk of developing osteoradionecrosis; therefore, dental management protocols prior to radiation may entail aggressive procedures such as extractions. Another explanation for this unanticipated caries prevalence is that 12 of 
the 19 studies were conducted in paediatric populations, who frequently consume highly cariogenic dietary supplements for weight maintenance or are taking sugar-rich medications.

The DMFT index was highest in patients who were post-radiation therapy compared to patients who were post-chemotherapy. This is not surprising since radiation damages salivary glands causing xerostomia, which is the most common toxicity associated with fractionated radiation therapy to the head and neck. ${ }^{2}$ Xerostomia decreases the oral $\mathrm{pH}$ and significantly increases the risk for development of plaque and dental caries. ${ }^{3}$ In addition, due to the lack of standardisation and wide ranges of time periods of DMFT data collection in included studies, it was not possible to examine trends of caries activity.

As for periodontal disease, the weighted prevalence of severe gingivitis from three included studies was $20.3 \%$. However, all three studies were conducted in patients undergoing chemotherapy. Additionally, four studies showed that patients who were postantineoplastic therapy had higher PI and GI than healthy controls. Attempts to describe periodontal health and periodontal disease beyond that of plaque and gingival indices in cancer patients were difficult in this review. This is primarily due to the various reporting methods and categorisations of periodontal disease.

In this review, the weighted prevalence of an odontogenic infection during chemotherapy was approximately 6\%. Despite the reported low prevalence of dental infections, there is evidence in the literature that these infections may become potentially life-threatening. The bacterial load of the mouth is greater than any other site of the body and, consequently, any changes to that environment can induce infection. As such, patients experiencing xerostomia are at particular risk of invasive gram-positive and gram-negative infections, as well as opportunistic infections with fungal organisms such as Candida. ${ }^{4}$ Oral candidiasis is one of the most common oral infections seen in association with xerostomia. ${ }^{5}$ Furthermore, bacterial and fungal colonisation of dental calculus, plaque, dental pulp and periodontal pockets comprises a reservoir of pathogenic and opportunistic organisms that may cause systemic infections during episodes of immune suppression resulting from cancer treatment.

The majority of the interventional studies in the review were conducted in patients who were post-head and neck radiotherapy, possibly because these individuals were thought to be at a much higher risk for the development of dental disease than post-chemotherapy patients. The main findings can be summarised in the following points:

- The use of fluoride products reduced caries in patients who were post-radiotherapy. The type of fluoride formulation or delivery system did not significantly influence caries activity.

- The use of chlorhexidine rinses reduced plaque scores and oral streptococcus mutans counts. This reduction was not seen with lactobacillus counts.

- Conventional glass ionomer restorations performed more poorly than resin-modified glass ionomer, composite resin and amalgam restorations in post-radiotherapy patients.
Furthermore, the authors recognised the scarcity of evidence pertaining to pre-cancer therapy dental clearance and the extent of disease requiring treatment. They also provided levels of evidence for preventive and management strategies in included studies. However, it is unclear how the authors evaluated the evidence, as they did not report the grading system they used. A variety of grading systems for evidence are currently in use. It is important for clinicians to be familiar with hierarchies of evidence and to be aware that evidence grading relates to the strength of the literature and not necessarily to clinical importance. In addition, it is essential to note that the hierarchy should primarily be dependent on the issue or question being researched. The Centre for Evidence-Based Medicine has recently published a very useful table to identify the different levels of evidence for different types of questions. ${ }^{6}$

This review underscores the current lack of clinical trials to evaluate the extent and severity of oral diseases associated with complications during cancer therapy. In addition, more studies are needed to determine the benefits of various types of pre-cancer therapy interventions on dental health. For example, there was only one article in the review on the benefits of amifostine, a cytoprotective agent that has been shown to reduce the incidence of dental caries associated with radiation-induced xerostomia. ${ }^{7}$ Similarly, future research is needed to verify the much-debated effectiveness of hyperbaric oxygen therapy in reducing the incidence of osteoradionecrosis in irradiated patients.

In conclusion, the incidence of oral complications in cancer patients can be reduced significantly when an aggressive approach to oral care is initiated prior to treatment. Primary preventive measures such as well-balanced nutritional intake, adequate oral hygiene and early detection and treatment of oral disease are important pre-treatment strategies. In the absence of conclusive evidence on pre-cancer therapy dental clearance, eradicating acute dental conditions as against eradicating both acute and chronic dental issues may be a more pragmatic option. Finally, patient education, supportive care and symptomatic medications remain important strategies in the management of patients experiencing oral complications of cancer therapy.

\section{Imad AI-Dakkak}

Centre for Evidence-based Dentistry, Oxford, UK

1. National Institutes of Health. Oral complications of cancer therapies: diagnosis, prevention, and treatment. National Institutes of Health Consensus Development Conference Statement, 17-19 April 1989; 7: 1-11.

2. Hensley ML, Schuchter LM, Lindley C et al. American Society of Clinical Oncology clinical practice guidelines for the use of chemotherapy and radiotherapy protectants. I Clin Oncol 1999; 17: 3333-3355.

3. American Dental Association. Public Resources: Oral health topics: Dry mouth. (Accessed November 12, 2010) http://www.ada.org/3014.aspx?currentTab=1

4. McDonald E, Marino C. Dry mouth: Diagnosing and treating its multiple causes. Geriatrics 1991; 46: 61-63.

5. Greenspan D. Xerostomia: diagnosis and management. Oncology 1996; 10: 7-11.

6. Levels of Evidence, Centre for Evidence-Based Medicine, June 2010 (Accessed November 12, 2010) http://www.cebm.net/index.aspx?o=5653

7. Rudat V, Meyer J, Momm F et al. Protective effect of amifostine on dental health after radiotherapy of the head and neck. Int I Radiat Oncol Biol Phys 2000; 48: 1339-1343.

Evidence-Based Dentistry (2011) 12, 15-16. doi:10.1038/sj.ebd.6400775 